DOI: https://doi.org/10.11144/Javeriana.umed61-4.caem

\title{
Consideraciones en la atención médica de pacientes con enfermedad mental e infección por COVID-19 en un hospital general
}

Considerations in the Medical Care of Patients with Mental Disorders and COVID-19 Infection in a General Hospital

\author{
Juana Yolanda Atuesta Fajardo \\ Médica psiquiatra. Psiquiatra de niños y adolescentes. \\ Jefa del servicio de Psiquiatría del Hospital de San José \\ de Bogotá, Colombia \\ Giovanny Armando Argoty Chamorro \\ Médico residente de segundo año de Psiquiatría, \\ Fundación Universitaria Ciencias de la Salud, Bogotá, \\ Colombia
}

a Autora de correspondencia: juanis765@gmail.com

Cómo citar: Atuesta Fajardo JY, Argoty Chamorro GA. Consideraciones en la atención médica de pacientes con enfermedad mental e infección por COVID-19 en un hospital general. Univ. Med. 2020;61(4). https://d oi.org/10.11144/Javeriana.umed61-4.caem

\section{RESUMEN}

Históricamente, los pacientes con enfermedad mental han sido un grupo poblacional de alta vulnerabilidad, más evidente en situaciones de emergencia sanitaria como lo es una pandemia. Por esta razón, en el artículo se describen las consideraciones específicas que puede enfrentar el personal de salud durante la atención de esta población en un hospital general, haciendo hincapié en las precauciones específicas y la necesidad de organización de grupos asistenciales que, además de tener una comunicación constante con el equipo de salud mental, deben establecer una adecuada relación terapéutica y estar preparados para responder a situaciones de urgencia, como lo es la agitación psicomotora. Palabras clave

trastornos mentales; pandemias; infecciones por coronavirus; hospitales generales.

\section{ABSTRACT}

Historically, patients with mental illness have been a highly vulnerable population group, which is most evident in health emergencies such as a pandemic. For this reason, this article describes specific considerations that health personnel may face during the care of this population in a general hospital. It is important to highlight the specific precautions and the need to organize healthcare groups that, in addition to having constant communication with the mental health team, must establish an adequate therapeutic relationship and be prepared to respond to emergency situations such as psychomotor agitation.

Keywords

mental disorders; pandemics; coronavirus infections; general; hospitals. 


\section{Introducción}

Los coronavirus son importantes patógenos en humanos y animales que surgen periódicamente en diferentes áreas del mundo y causan en los humanos infección respiratoria aguda (1). A finales de 2019, se identificó un nuevo coronavirus como agente causal de un grupo de casos de neumonía en Wuhan (capital de la provincia de Hubei), el cual se extendió rápidamente en China y, posteriormente, por todo el mundo. En consecuencia, en marzo de 2020, la Organización Mundial de la Salud reconoció la enfermedad por coronavirus 2019 (COVID-19) como una pandemia mundial $(1,2)$.

En Colombia, el primer caso se registró el 6 de marzo de 2020 (3) y, como se había previsto, desencadenó una serie de medidas nacionales y locales, así como la implementación de las recomendaciones dadas por la Organización Mundial de la Salud para proteger a los trabajadores sanitarios, entre las cuales se encuentran los protocolos de atención y el uso de elementos de protección personal (4); pero, como bien lo describe Albert Camus en el libro La peste (1947): "Ha habido en el mundo tantas pestes como guerras. Y, aun así, las pestes y las guerras toman a todo el mundo desprevenido". Es decir, durante el transcurso de la pandemia se ha requerido planificar e implementar nuevas y variadas estrategias que respondan al impacto y a las exigencias de los distintos escenarios que plantea la infección por COVID-19 en el ambiente hospitalario (5).

A estas exigencias se ha asociado la sobrecarga laboral que enfrenta el equipo médico en un hospital general. En este contexto, los médicos psiquiatras no solo están a cargo de la atención de las necesidades emocionales de los pacientes, sino también del personal de salud, en función de catalizar el temor y la ansiedad relacionadas con las circunstancias actuales. Además, deben enfrentar diferentes barreras para que sus pacientes accedan a los servicios de salud, y dicho contexto permite reconocer que las personas con un trastorno mental o del comportamiento aún cargan con un estigma social importante, asociado a discriminación y exclusión (6), que lleva a pensar a muchos médicos no psiquiatras que el único sitio donde deben ser tratados los pacientes con enfermedad psiquiátrica es en la unidad de salud mental. Como si desconocieran que, en la mayoría de estos lugares, no se cuenta con la infraestructura ni con el personal para garantizar atención integral a otra condición mórbida no mental y que el tener patología psiquiátrica no puede ser impedimento para recibir atención médica intramural en un hospital general.

El objetivo del artículo es plantear una propuesta de atención a pacientes con trastornos mentales e infección por COVID-19 en el escenario de un hospital general, dado que la percepción negativa hacia estos pacientes dificultaría su adecuada atención (7). De esta forma, se señalan los principales elementos que se buscan impactar, como es el caso de la organización de grupos asistenciales que, además de tener una comunicación constante con el equipo de salud mental, deben establecer una adecuada relación terapéutica y estar preparados para responder a las diferentes exigencias que se pueden presentar.

\section{Propuesta de atención médica para pacientes con trastornos mentales e infección por COVID-19 en un hospital general}

El número de pacientes con trastornos mentales que busca atención en salud durante la pandemia ha aumentado; por ende, los retos para los psiquiatras y el personal de salud con los pacientes hospitalizados por infección por COVID-19 y trastornos mentales son cada vez mayores $(8,9)$. Este fenómeno se ha asociado a los diversos factores estresantes que empeoran los síntomas psiquiátricos, como las restricciones propias del aislamiento como estrategia principal de las políticas en salud, que buscan reducir la tasa de transmisión de la infección por COVID-19 (10), lo que limita el seguimiento ambulatorio (5).

Esta nueva dinámica ha modificado las relaciones sociales, en especial con aquellos 
grupos poblacionales que se consideran vulnerables, incluida la población con enfermedad mental grave (10), término que se ha utilizado para describir a pacientes con esquizofrenia o trastorno afectivo bipolar. Actualmente, este término no solo se asocia con las patologías descritas, sino que es un concepto más amplio que engloba aquellas enfermedades mentales que generan un deterioro funcional grave (11).

Dentro de la vulnerabilidad de esta población, cabe resaltar el alto riesgo de contagio secundario a condiciones de hacinamiento, ya que la mayoría se encuentra viviendo en entornos colectivos como unidades de salud mental, albergues o cárceles, resultado de la progresión crónica de su enfermedad o la desventaja social (10).

Por otra parte, si estas patologías se asocian con una afectación cognitiva, pueden constituirse en un impedimento para darles de alta a los pacientes y que cumplan su cuarentena en casa, ya que la baja conciencia del riesgo dificulta el seguimiento de recomendaciones para el control de infección por COVID-19. Además, debe tenerse en cuenta que esta población presenta altas tasas de obesidad, enfermedad cardiovascular y enfermedad pulmonar obstructiva asociada $(5,10)$.

La importancia de los factores descritos se ha evidenciado en los diferentes reportes presentados en países que ya se encuentran en descenso de su curva de contagio como China, donde el Centro de Salud Mental de Wuhan informó que aproximadamente 50 pacientes hospitalizados y 30 miembros del personal médico presentaron prueba de COVID-19 positiva (12). De igual forma, en el hospital psiquiátrico de Corea del Sur informó que 101 de 103 hospitalizados contrajeron COVID-19 durante un brote, de los cuales murieron 7 finalmente (13). En Colombia, una clínica de salud mental comunicó un brote de coronavirus, con 45 casos entre trabajadores y pacientes, que dio como resultado una víctima fatal (14). El Ministerio de Salud y Protección Social, en sus boletines de prensa, ha informado un mayor número de llamadas a las líneas de atención en salud mental (9).

De esta manera, se entiende que los pacientes con trastornos psiquiátricos son vulnerables no solo por su enfermedad mental, sino también por las repercusiones de la COVID-19; además, es innegable mencionar las dificultades administrativas, estructurales y sociales que podrían entorpecer aún más la prestación del servicio en salud $(5,6)$.

Por ello, es necesario que en el hospital general se plateen ajustes operativos que garanticen no solo el aislamiento del paciente por el riesgo de contagio, sino también la continuidad del manejo psicofarmacológico, el seguimiento intrahospitalario por parte del servicio de psiquiatría y el establecimiento de estrategias que posibiliten la atención a las necesidades psicológicas que pueda presentar. Dentro de esta experiencia, se resalta que un liderazgo organizado (5) y una comunicación efectiva son los pilares para instaurar programas de atención eficientes dentro de cada uno de los grupos involucrados en el cuidado del paciente (personal médico, de enfermería, trabajo social y psicología) que no es más que garantizar una atención médica con dignidad, lo que significa llevar a la práctica el concepto de humanización en salud (15).

En conclusión, los diferentes factores nombrados han impulsado a proponer el desarrollo de unidades psiquiátricas positivas para COVID-19 (13). Por ende, tomando estas recomendaciones y modificándolas al entorno hospitalario y según la experiencia adquirida, se enumeran: precauciones específicas, paciente hospitalizado, atención psicomotora, consideraciones éticas, proceso de alta, asesoría al acompañante del paciente hospitalizado y cuidado de la salud mental del personal asistencial.

\section{Precauciones específicas}

El equipo de salud debe contar con los medios de protección requeridos (4), así como seguir las recomendaciones del lavado 
de manos y desinfección de objetos. Sin olvidar la importancia del automonitoreo para detectar sintomatología respiratoria acompañada de fiebre, de modo que se pueda realizar una intervención temprana, para evitar la propagación del virus (5).

Por consiguiente, el hospital requerirá una planificación espacial para establecer áreas que permitan ponerse y quitarse el equipo de protección personal apropiado, para evitar la contaminación de las zonas de trabajo que no son COVID-19 (13). Cabe resaltar que estos lugares serán claramente identificados y demarcados.

No se puede negar que, en el país, muchas de las instituciones hospitalarias no cuentan con la infraestructura adecuada para hacerle frente a la crisis sanitaria y, en muchos casos, tampoco con los elementos adecuados de bioseguridad para el personal sanitario (16). En consecuencia, se les hace un llamado a las directivas de las diferentes entidades territoriales para que adecúen los espacios mencionados, así como para que diseñen estrategias que intensifiquen las medidas de bioseguridad para el talento humano, con el fin de reducir la exposición del personal de salud.

Ahora bien, en favor de incrementar las medidas de seguridad contra infecciones respiratorias, es importante promover el distanciamiento social, estrategia que reduce la exposición externa adicional, al implementar las restricciones de visitas para miembros de la familia, empleados no esenciales y estudiantes, sin que esto se traduzca en un aislamiento completo del paciente. En efecto, el servicio tratante deberá buscar los medios adecuados para darle continuidad a la comunicación entre el paciente y su familia (5).

\section{Paciente hospitalizado}

No se limitará el acceso de los pacientes con enfermedades mentales e infección por COVID-19 que requieran hospitalización. Así mismo, es obligatorio un abordaje empático por parte del personal de salud, asociado a una monitorización cercana por el riesgo de auto o heteroagresión (5). Es clave el fortalecimiento de la alianza terapéutica, dado que la mayoría de los pacientes psiquiátricos que han tenido que ser aislados en hospital general por infección por COVID-19 presentaron exacerbación de su angustia psicológica (12).

En consecuencia, cada grupo de trabajo que tenga a cargo estos pacientes, tendrá como mínimo la identificación de líderes, quienes tendrán comunicación constante con el área de salud mental del hospital, para abordar las situaciones que requieran una solución inmediata (5). Además, por medio de reuniones constantes de estos líderes con los médicos psiquiatras, se discutirá información, se implementarán decisiones y se abordarán los desafíos clínicos $(5,12)$; así mismo, ello permitirá la catarsis de los miembros del equipo para identificar las defensas individuales que puedan llevar a que la atención que se da a los pacientes con patología psiquiátrica e infección por COVID-19 no es la adecuada y brindar las herramientas para mejorarla. De no lograr resultados favorables, podría considerarse el cambio de personal asistencial en esa área.

\section{Agitación psicomotora}

La agitación psicomotora es una urgencia médica, definida como un estado de marcada excitación mental que se acompaña de un aumento inadecuado de la actividad motora (17). Por ello, su atención involucra no solo al equipo de salud mental, sino también al servicio tratante, porque es esencial una adecuada respuesta que cuide de la integridad del paciente, sus familiares, el personal sanitario y el entorno en general $(17,18)$.

Por ende, el anticipar estas situaciones y desarrollar protocolos de respuesta con su respectiva socialización para abordar al paciente agitado, permitirá tener claras las indicaciones de contención verbal, física y farmacológica, así como el papel de cada uno de los integrantes del equipo durante el evento $(13,17)$, haciendo hincapié en que cualquier médico y personal de enfermería deben estar en capacidad de 
controlar la situación, dado el riesgo de auto y heteroagresión. Con la capacitación del trabajador de la salud que tiene a cargo estas áreas habrá un reconocimiento temprano y un manejo adecuado de la agitación psicomotora (19).

\section{Consideraciones éticas}

Las connotaciones éticas que se movilizan en la relación médico-paciente se suelen reflejar en diferentes modelos, como el paternalista, el autonomista, el interpretativo y el deliberativo, en los que prevalece algún principio bioético ligado (20). En el caso de los trastornos mentales graves, en los cuales destaca la vulnerabilidad ante el contagio por COVID-19, producto de la desventaja social y el mayor riesgo de complicaciones, sobresale el modelo médicopaciente de tipo interpretativo $(10,20)$, en el que en favor de la beneficencia sobre la autonomía se indicaría una hospitalización involuntaria.

Ya en el marco legislativo nacional se cuenta con la Ley 1306 del 2009, en la cual se describe que "los pacientes con discapacidad mental absoluta solamente podrán internarse en clínicas o establecimientos especializados por urgencia calificada por el médico tratante" (17). De ahí que se le otorgue al servicio médico el actuar en consecuencia a la seguridad y bienestar del paciente; análisis que debe ser detallado y descrito en la historia clínica.

\section{Proceso de alta}

Según la evolución clínica y las indicaciones médicas dadas por los servicios tratantes, en consenso se definirá la continuidad del tratamiento del paciente, ya sea en una unidad psiquiátrica o cuidados en casa, teniendo en cuenta la capacidad de la familia y pacientes para cumplir con la cuarentena si fuera necesario (13). Dentro de la planificación del alta se deben brindar recomendaciones generales, signos de alarma, seguimiento y, en lo posible, disponibilidad de teleconsulta.
Asesoría al acompañante del paciente hospitalizado

El médico psiquiatra, junto con un equipo multidisciplinario, conformado por la especialidad tratante, enfermería, trabajo social y psicología, debe procurar la prestación de una atención integral. Además, en el proceso de hospitalización del paciente con enfermedad mental y COVID-19, se deberá involucrar de forma activa a la familia para detectar elementos que entorpezcan la posterior recuperación del paciente en casa, dado que la pandemia en la población general combina factores como la ansiedad por la salud, el miedo a la muerte y el miedo a perder seres queridos, que, sumados al impacto social, pueden desencadenar o reactivar trastornos mentales graves. Son importantes las intervenciones dirigidas a los acompañantes que incluyan psicoeducación y manejo de los síntomas comunes del estrés, abordando técnicas de relajación y promoción de estilos de vida saludables, que generen un impacto positivo (21).

\section{Cuidado de la salud mental del personal asistencial}

Los profesionales de la salud, por su mayor exposición a las diferentes consecuencias psicosociales de la pandemia, están propensos a desarrollar consecuencias importantes en su salud mental. Es primordial identificar factores estresantes y establecer estrategias que permitan el cuidado (22).

\section{Conclusiones}

Es necesario tener claridad en estos conceptos y herramientas para lograr organizaciones de alta efectividad, cuyo objetivo sea mejorar la asistencia clínica de esta población específica, que involucra el aprendizaje continuo y, de esta forma, diseñar protocolos específicos que minimicen la estigmatización y la aplicación inadecuada de los procesos asistenciales.

Los pacientes con trastornos psiquiátricos siguen siendo un grupo vulnerable que requiere 
mayor atención y respeto, particularmente durante el brote de COVID-19 y después de este. La enfermedad mental es un antecedente médico como cualquier otro y no debe ser motivo de estigmatización; por el contrario, su aceptación y entendimiento es garante de la atención integral que todo paciente requiere.

Las instituciones que atienden a pacientes con infección por COVID-19 y que además tienen enfermedad mental no deben restringir su acceso. En cambio, es esencial que el personal asistencial en general y no solo el personal de salud mental tenga un trato empático para fortalecer la alianza terapéutica.

Se debe capacitar al personal de salud en general, para la evaluación, el abordaje y la atención del paciente con agitación psicomotora, con el fin que tenga un manejo oportuno y se minimice el riesgo de daño propio o a terceros.

\section{Referencias}

1. McIntosh K, Hirsch MS, Bloom A. Coronavirus disease 2019 (COVID-19) [internet]. Disponible en: https://www.u ptodate com/contents/coronavirusdisease-2019-covid-19-epidemiologyvirology-clinical-features-diagnosisand-prevention

2. World Health Organization. WHO director-general's opening remarks at the media briefing on COVID-19. 11 de marzo de 2020 [internet]. Ginebra: WHO; 2020. Disponible en: https://www.who.int/dg/speeches/d etail/who-director-general-s-opening-r emarks-at-the-media-briefing-on-covi d-19.--11-march-2020

3. Semana. Coronavirus en Colombia: primer caso confirmado [internet]. Bogotá; 3 de junio de 2020. Disponible en: https://www.semana.com/nacion/a rticulo/coronavirus-en-colombia-prim er-caso-confirmado/655252

4. Feng S, Shen C, Xia N, Song W, Fan M, Cowling BJ. Rational use of face masks in the COVID-19 pandemic. Lancet Resp Med. 2020;8(5):434-6.

5. Li L. Challenges and priorities in responding to COVID-19 in inpatient psychiatry. Psychiatr Serv. 2020;71(6):624-6. https://doi.org/10.1 176/appi.ps.202000166

6. Ahmedani BK. Mental health stigma: society, individuals, and the profession. J Soc Work Values Ethics. 2011;8(2):41-416.

7. Giandinoto JA, Stephenson J, Edward KL. General hospital health professionals' attitudes and perceived dangerousness towards patients with comorbid mental and physical health conditions: Systematic review and meta-analysis. Int J Ment Health Nurs. 2018;27(3):942-55.

8. Czeisler MÉ, Lane RI, Petrosky E, Wiley JF, Christensen A, Njai R, et al. Mental health, substance use, and suicidal ideation during the COVID-19 pandemic - United States, June 24-30, 2020. MMWR Morb Mortal Wkly Rep. 2020 Aug 14;69(32):1049-57. https://d oi.org/10.15585/mmwr.mm6932a1

9. Ministerio de Salud y Protección Social. Línea 192 ya ha atendido 2.100 llamadas para atención en salud mental [internet]. Bogotá; 16 de mayo de 2020. Disponible en: https://www.minsalud.gov.co/Pagi nas/Linea-192-ya-ha-atendido-2.100-11 amadas-para-atencion-en-salud-menta l.aspx

10. Shinn AK, Viron M. Perspectives on the COVID-19 pandemic and individuals with serious mental illness. J Clin Psychiatry. 2020;81(3). https://d oi.org/10.4088/JCP.20com13412

11. World Health Organization. Helping people with severe mental disorders live longer and healthier lives. Ginebra; 2017. 
12. Li S, Zhang Y. Mental healthcare for psychiatric inpatients during the COVID-19 epidemic. Gen Psychiatr. 2020;33(2):e100216. https://doi.org/1 0.1136/gpsych-2020-100216

13. Cheung E, Strouse T, Li L. Planning for a psychiatric COVID-19-positive unit. MDedge [internet]. 15 de mayo de 2020. Disponible en: https://www.m dedge.com/psychiatry/article/222331/c oronavirus-updates/planning-psychiatr ic-covid-19-positive-unit

14. Cabrera D. Confirman muerte de auxiliar de enfermería de Clínica Ntra. Señora de La Paz por coronavirus. Lafm [internet]. 7 de mayo de 2020. Disponible en: https://www.lafm.com.co/bogota/c onfirman-muerte-de-auxiliar-de-enfer meria-de-clinica-ntra-senora-de-la-paz -por-coronavirus

15. Bermejo Higuera JC. Humanización de la atención [internet]. Bogotá; 2016. Disponible en: http://www.acreditacionensalud.or g.co/ea/Paginas/HumAte.aspx.

16. Lizarazo Riaño SC. iEstá preparado el sistema de salud colombiano para enfrentar el COVID-19? [internet]. Sociedad Colombiana de Anestesiología y Reanimación; 18 de marzo de 2020. Disponible en: https://scare.org.co/noticias/esta-pr eparado-el-sistema-de-salud-colombia no-para-enfrentar-el-covid-19/

17. Escobar-Córdoba F, Suárez-Beltrán MF. Abordaje clínico del paciente violento en atención primaria. Univ Méd. 2011;52(4):421-30. https://doi.or g/10.11144/Javeriana.umed52-4.acpv

18. Rossi J, Swan MC, Isaacs ED. The violent or agitated patient. Emerg Med Clin North Am. 2010;28(1):235-56. https://doi.org/10. 1016/j.emc.2009.10.006
19. Nieto R, Iribarne C, Droguett M, Brucher R, Fuentes C, Oliva P. Urgencias psiquiátricas en el hospital general. Rev Méd Clín Las Condes. 2017;28:914-22. https://doi.org/10.101 6/j.rmclc.2017.09.004

20. Mendoza A. La relación médico paciente: consideraciones bioéticas. Rev Peru Ginecol Obstet [internet]. 2017;63(4):555-64. Disponible en: http://www.scielo.org.p e/scielo.php?script $=$ sci_arttext\&pid $=$ S2304-51322017000400007

21. Kavoor AR. COVID-19 in people with mental illness: challenges and vulnerabilities. Asian J Psychiatr. 2020;51:102051. https://doi.org/10.10 16/j.ajp.2020.102051.

22. Téllez-Vargas J. La salud mental del médico durante la pandemia. Eurofarma; 2020. p. 9.

\section{Notas}

Conflicto de intereses: Los autores declaran que no presentan conflicto de intereses con la publicación del artículo y que no se contó con ninguna fuente de financiación para su elaboración. 